This is a self-archived - parallel published version of this article in the publication archive of the University of Vaasa. It might differ from the original.

\title{
A New Control Strategy for Harmonic Mitigation Using Open UPQC in Modern LV Networks
}

Author(s): Faranda, R.; Kazemi-Robati, E.; Sepasian, M. S.; Akkala K.; Hafezi, H.

Title: $\quad$ A New Control Strategy for Harmonic Mitigation Using Open UPQC in Modern LV Networks

Year: $\quad 2019$

Version: Accepted manuscript

Copyright (C)2019 IEEE. Personal use of this material is permitted. Permission from IEEE must be obtained for all other uses, in any current or future media, including reprinting/republishing this material for advertising or promotional purposes, creating new collective works, for resale or redistribution to servers or lists, or reuse of any copyrighted component of this work in other works.

Please cite the original version:

Faranda, R., Kazemi-Robati, E., Sepasian, M. S., Akkala K. \& Hafezi, H. (2019). A New Control Strategy for Harmonic Mitigation Using Open UPQC in Modern LV Networks. In: 2019 IEEE PES Innovative Smart Grid Technologies Europe (ISGT-Europe), 1-5. https://doi.org/10.1109/ISGTEurope.2019.8905688 


\title{
A New Control Strategy for Harmonic Mitigation Using Open UPQC in Modern LV Networks
}

\author{
R. Faranda ${ }^{1}$, E. Kazemi-Robati ${ }^{2}$, M. S. Sepasian ${ }^{2}$, K. Akkala ${ }^{1}$, H. Hafezi ${ }^{3}$ \\ ${ }^{1}$ Department of Energy, Politecnico di Milano, Milano, Italy \\ ${ }^{2}$ Department of Electrical Engineering, Shahid Beheshti University, Tehran, Iran \\ ${ }^{3}$ School of Technology and Innovations, University of Vaasa, Vaasa, Finland \\ Email: roberto.faranda@polimi.it
}

\begin{abstract}
The higher penetration of both renewable generation units and nonlinear loads is leading to higher levels of harmonics in microgrids and modern distribution systems. Accordingly, the operation of the sensitive loads in the grid can be affected by the low quality of the power delivered to the customers. Moreover, the higher harmonic levels lead to an increase in losses and lower energy efficiency in the grid. Hence, the harmonics in the grid are regarded as a great challenge by both the customers and Distribution System Operators (DSOs). Accordingly, the researchers have widely focused on minimizing the harmonics of the modern grids using Custom Power Devices (CPDs) in recently published papers. Among the devices incorporated in previous studies, the Open-UPQC is found as an effective and applicable solution for power quality improvement in microgrids and modern distribution systems which can provide benefits for both customers and DSOs. In this paper, a new coordination strategy is proposed for the Open-UPQC to provide the highest harmonic elimination capability besides the voltage improvement in the grid. In order to evaluate the effectiveness of the proposed method, the control strategy is applied to a sample modern distribution system which is formed by adding harmonic-injecting loads to a grid with real data. The results of simulations verified that applying the proposed control strategy to the Open-UPQC provides the effective harmonic reduction in the grid by using the improved coordination of the shunt units.
\end{abstract}

Keywords-Open-UPQC, LV distribution systems, microgrid, ancillary service, power quality, real-time control.

\section{INTRODUCTION}

The harmonic currents in the distribution systems can negatively affect both the facilities in the grid and the customer devices [1], [2]. Accordingly, there are some limitations introduced by IEEE and IEC for the acceptable harmonic levels in the grid [3]. Such standards introduced for the quality of delivered power can provide the possibility of normal operation for the connected devices and can also guarantee the anticipated lifetime of facilities specified by the manufacturers [4]. The importance of providing such specifications for the delivered power increases when there are so many sensitive devices connected to modern grids. Data banks, computers and control systems are some of these sensitive and important devices which are used in industrial, business, domestic and governmental levels of the grid and should be supplied with respect to the specified standards [4].

On the other hand, providing the levels of power quality requested by the customers is getting more complicated due to the higher penetration of the new elements of the modern grids. From a load point of view, the higher penetration of Power Electronic (PE) converters can affect the quality of the power in the grid. On the other hand, form a generation point of view, providing a higher portion of the supplied power by the PE-interfaced DG units affects the power quality in the grid. Hence, it is clear that the quality of power is negatively affected by the new trends in both sides of generation and consumption.

Due to the given description, there are lots of studies published on power quality improvement in modern distribution systems using Custom Power Devices (CPDs) during recent years [5]-[9]. Accordingly, different topologies and control systems are proposed by the researchers to provide an acceptable improvement in the quality of the power delivered to the end users. DVR [6], DSTATCOM [7] and UPQC [8] are some of the main devices incorporated in power quality improvement in the studied grids.

Among the proposed solutions, UPQC can provide the most effective improvement due to the presence of both series and shunt units in the topology of the device. On the other hand, such a complicated and expensive topology decreases the applicability of the device. Accordingly, the idea of coordinated control of a series unit with the existing shunt units in the grid has been proposed as Open-UPQC in [10]. Hence, by adding a series unit to the grid and using the available capacity of the shunt units owned by the customers, all of the capabilities of UPQC can be provided with much lower investment cost.

The cooperation between series and the shunt units of the Open-UPQC using the available communication systems can provide many benefits like coordinated voltage improvement in the grids [10]. According to the strategy proposed in [10], by reactive power support from the shunt units of the OpenUPQC, the series unit can provide the desired voltage at PCC while a wider range of substation voltage deviations is covered. Hence, without any additional cost, effective power quality improvement in the grid can be provided by such coordination.

In addition to the previously proposed strategy for voltage 
improvement using Open-UPQC, there is the possibility of the cooperation between series and shunt units for harmonic elimination in modern distribution systems. Accordingly, using a supplementary control strategy, the harmonic currents measured in the substation can be compensated using the shunt units of the grid. In other words, the harmonic currents of the nonlinear loads which are not compensated locally can be supplied through neighbor shunt units with respect to measurements in the substation. Hence, the harmonic currents injected to the other parts of the grid are minimized and the lowest THD levels are provided in the substation.

Due to the given description, in this paper, a supplementary control strategy is proposed for Open-UPQC for coordinated harmonic elimination in Modern LV distribution systems. Accordingly, the harmonic currents measured by the series unit will be shared among the available shunt units in the grid. Hence, besides the voltage improvement using the device, the harmonic currents can be minimized using a simple coordination strategy in the grid.

The rest of this paper is organized as follows. State of art review on more common $\mathrm{PE}$ devices in modern grids is provided in section II. The coordination strategy for harmonic elimination is proposed in section III. A description of the studied grid is provided in section IV. The simulation results are given and analyzed in section $\mathrm{V}$ and the paper is concluded in section VI.

\section{PE DEVICE IN MODERN GRIDS}

As described in the previous section, lots of the devices in modern distribution systems are connected to the grid through PE interfaces. Accordingly, PE-interfaced devices are considered as both origin and potential solution for harmonics in the grids.

From a harmonic-injection point of view, the higher penetration of PE-interfaced DGs and storage systems besides the nonlinear loads of the grid leads to lower levels of power quality in the grid. On the other hand, the several types of CPDs proposed by researchers are found as effective solutions for harmonic minimization in the grids.

The CPDs introduced by the researchers can be primarily categorized to shunt and series devices. Furthermore, an improved power quality enhancement capability can be provided by a combination of these two common structures. A brief review of the different topologies proposed for the devices is given in the following subsections.

\section{A. Series Conditioner}

The diagram of the series conditioner which is called Dynamic Voltage Conditioner (DVC) is given in Fig. 1.

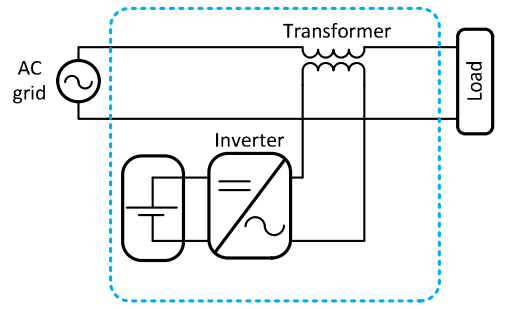

Fig. 1. Diagram of a DVC.

This device is designed to be connected between the grid and the loads and it is capable to compensate voltage sags/swells, flickers and long term voltage problems. The storage system shown in this figure is a set of capacitors used for reactive power injection and the converter works as a voltage source in the grid. It should be noted that DVC needs a very small power source (capacitors) connected to the DC link for the transients.

\section{B. Uninterruptible Power Supply (UPS)}

The diagram of an online UPS system is given in Fig. 2. As shown in this figure, the power from the main grid is initially converted to DC and the load is supplied through the $\mathrm{DC} / \mathrm{AC}$ inverter. Accordingly, the load can be supplied by variable voltage and frequency. Moreover, the desired power factor on the grid side can be provided using an active AC/DC converter (grid side). The power source (RES and battery) is connected to the DC link of the device and can supply the load in case of grid failure.

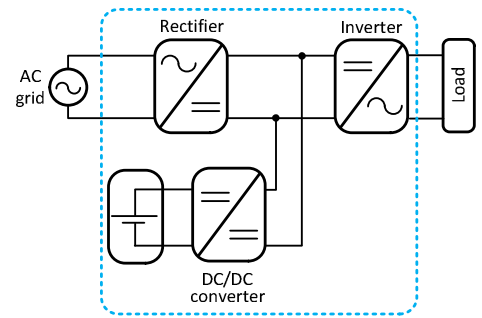

Fig. 2. Diagram of a double-conversion online UPS.

\section{Shunt Conditioner}

Diagram of a shunt conditioner is given in Fig. 3. As shown in the figure, this device is connected in parallel with the load or the grid. It can also be supported using a storage system. In this case, the device can be considered as an offline UPS system. This device can supply the load from the storage system through the converter while the frequency is imposed by the grid. If the power source is replaced with a set of capacitors, the active power supply cannot be accomplished and the device can be controlled to work as a DSTATCOM.

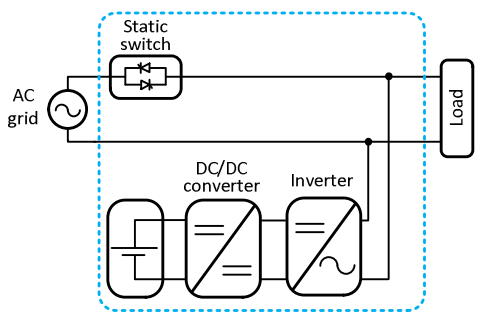

Fig. 3. Diagram of a shunt conditioner.

\section{UPQC}

As the defects of the series and shunt units can be covered in a combinational use of the both configurations, researchers have proposed the UPQC as a comprehensive solution to the both current and voltage quality problems in the grid. The diagram of a sample UPQC is given in Fig. 4. As shown in the figure, there is a shared DC link for both series and shunt units of the UPQC. This device also comprises two DC/AC converters, a static switch, a coupling transformer and a $\mathrm{DC} / \mathrm{DC}$ converter for the power source. Accordingly, although the device provides many services to improve the power quality, the higher cost due to the different elements in 
the UPQC configuration decreases the applicability of the device in real-world applications.

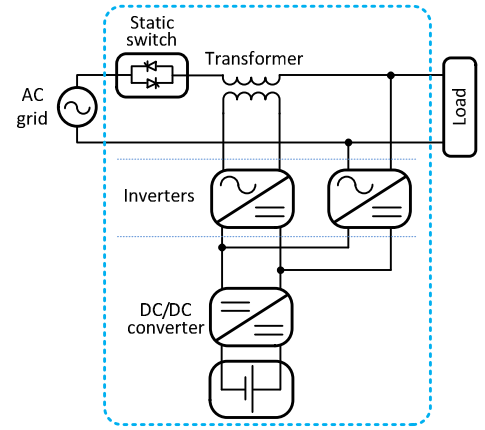

Fig. 4. Diagram of a UPQC.

\section{E. Open-UPQC}

Due to the presence of several shunt units in the grid, the coordinated control of these devices and the series unit in the substation of a distribution system can provide all the benefits of UPQC with considerable cost decrease. Accordingly, the idea of Open-UPQC is defined in [10], [11] and the sample diagram of the device with one shunt unit is given in Fig. 5 . In real-world applications, the device comprises several shunt units close to user points which are working in coordination with the series unit. Accordingly, the reliable communication system which can be provided in smart grids and modern distribution systems plays a key role in the implementation of the Open-UPQC idea. By such a strategy, several benefits can be provided for the users and DSOs.

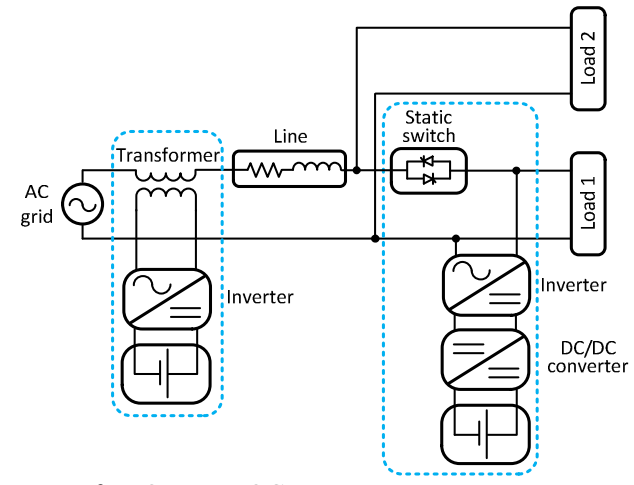

Fig. 5. Diagram of an Open-UPQC.

\section{PROPOSED STRATEGY FOR COORDINATED HARMONIC MITIGATION USING OPEN-UPQC}

The structure of the connection between all the units of Open-UPQC is given in Fig. 6. According to the figure and the description given in Section II, it is clear that there is the potential for providing whole-grid harmonic mitigation by the use of the communication between the series and the several shunt units of Open-UPQC. Due to the working principles of the Open-UPQC, each shunt unit is responsible for peak shaving, reactive power compensation and harmonic elimination of its local load. Moreover, in the case of improving the grid voltage, the series unit sends a request of extra reactive power to all of the shunt units. Hence, by a centralized control strategy, each shunt unit can enhance the voltage improvement capability of the series unit through a change in its reactive power reference [12].

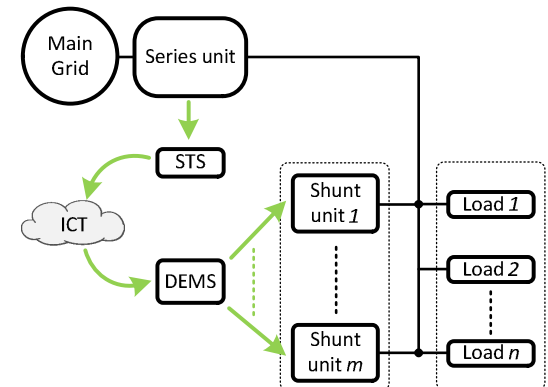

Fig. 6. The coordinated control of the series and the several shunt units of the Open-UPQC

As the harmonic currents can be measured in the substation of the distribution system, a similar coordination method can be applied as a supplementary control strategy for harmonic minimization. Accordingly, based on the harmonic measurement in the substation, each shunt unit is called by the central control system for the compensation of a part of harmonic currents in the grid. Such a request is sent to each shunt unit as phase angle and magnitude for any harmonic current order.

In this method, the measured substation line current is analyzed for specified harmonic orders and the magnitude and phase of the related harmonic currents are extracted. In order to compensate the measured harmonic in the grid using an active filtering method, a current should be generated by the shunt units in opposite direction. Accordingly, the measured current angle for the harmonic order should be directly sent to the shunt units without any modification. On the other hand, the current magnitude should be shared between the units. Due to the presence of the measurement system on the substation, the reasonable solution is the equal sharing of the measured harmonic current magnitude by the all units. Accordingly, each of the $\mathrm{M}$ shunt units prepares $1 / \mathrm{M}$ of the magnitude of the harmonic current. Hence, the coordinated control strategy for harmonic minimization is implemented in the grid without any information about the location of the nonlinear loads.

In order to apply the control method to Open-UPQC, there are some considerations related to practical aspects which should be taken into account. Accordingly, communication system limits is one of the important points which should be considered. Therefore, there should be a reasonable time interval between the commands sent to the shunt units. Furthermore, a time-period should be considered between the sent signals and the ones received by the shunt units as the delay of the communication system.

Due to delay in the control strategy and in order to avoid the continuous oscillation of the control signals, there should be a dead-band in updating the current reference of the shunt units based on the measured THD in the substation. The operation of the controller based on the dead-band can be described with respect to Fig. 7 where in the starting point "a", the measured THD is higher than the allowed limit. So the THD will be decreased to "b", "c", "d" and finally to the "e" point using the control method. In this point, the control output is maintained constant and equal to the last calculated one. When the THD increases to values higher than the upper boundary, a new control output will be calculated after resetting the controller. 


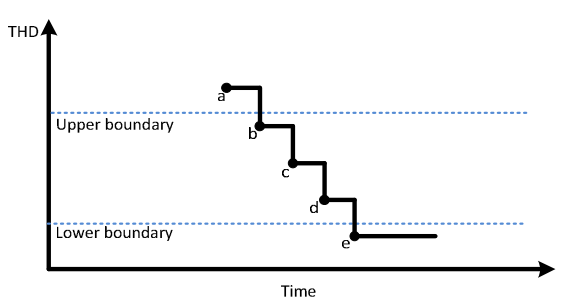

Fig. 7. Dead-band for the proposed controller

\section{A. Structure of the signals}

According to the measured harmonics, the coordination strategy is applied through telecommunication signals which are sent to the shunt units. As the harmonic currents are equally shared between the shunt units, the signals sent to all units are the same. The structure of the signal is shown in Fig. 8. In this figure, $\left|\mathrm{I}_{\mathrm{sh}, \mathrm{hCC}}^{\mathrm{h}}\right|$ and $\angle \mathrm{I}_{\mathrm{sh}, \mathrm{CC}}^{\mathrm{h}}$ represent the magnitude and the phase of the $\mathrm{h}^{\text {th }}$ order harmonic current requested by the central controller to be produced by the shunt unit.

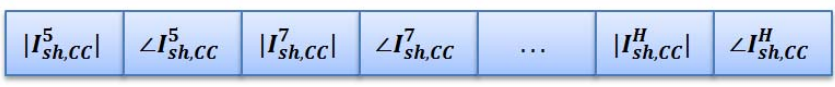

Fig. 8. The structure of the signal sent to shunt units

\section{NETWORK, LOAD AND DISTURBANCES}

In order to investigate the performance of the proposed control method, it is applied to a simple single phase distribution system which is formed using the real measured data. The single line diagram of the grid is given in Fig. 9, in which $L_{1}, L_{2}$ and $L_{3}$ are the 200-meter distribution lines of the feeder. The cable data of the LV network is also given in Table I.

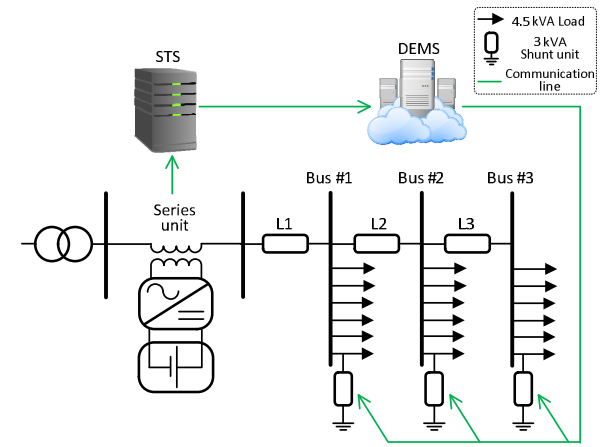

Fig. 9. Single line diagram of the studied grid.

TABLE I. SPECIFICATIONS OF THE CABLES USED IN THE STUDIED NETWORK

\begin{tabular}{|c|c|c|c|}
\hline $\begin{array}{c}\text { Nominal section } \\
\left(\mathbf{m m}^{2}\right)\end{array}$ & $\begin{array}{c}\mathbf{R} \\
(\mathbf{m} \boldsymbol{\Omega} / \mathbf{m})\end{array}$ & $\begin{array}{c}\mathbf{X} \\
(\mathbf{m} \boldsymbol{\Omega} / \mathbf{m})\end{array}$ & $\begin{array}{c}\text { Current carrying } \\
\text { capacity } \mathbf{( A )}\end{array}$ \\
\hline 185 & 0.123 & 0.0908 & 545 \\
\hline
\end{tabular}

As there is no meter for harmonic currents in the real grid and the measured data comprise active power, reactive power, RMS voltage and RMS current, some load modifications should be applied to the system. Accordingly, for the study accomplished in this paper, some nonlinear loads are added to the different busses of the grid. These single phase loads are considered as the sources of pure 5th and 7th harmonic currents. The 5th and 7th order harmonic current magnitudes are selected to be $15 \%$ and $10 \%$ of the fundamental current due to the linear loads, respectively.
The substation bus current waveform for a sample case is given in Fig. 10 in which the THD is $10.28 \%$. For the studied case, the active and reactive power of the linear loads are given in Table II. The values of the table are provided from the real measurements in the grid. It should be noted that due to the Fig. 9, there is one load point in each bus which is equipped with a shunt unit. Hence, regardless of the central control strategy, the harmonic current of this load is compensated independently by its shunt unit.

TABLE II. LOAD DATA FOR THE STUDIED CASE

\begin{tabular}{|c|c|c|c|}
\hline Variable & Bus \#1 & Bus \#2 & Bus \#3 \\
\hline$P_{\text {Linear }}(\mathrm{kW})$ & 2.17 & 9.65 & 3.33 \\
\hline$Q_{\text {Linear }}(\mathrm{kVAr})$ & 1.07 & 0.91 & 1.18 \\
\hline
\end{tabular}

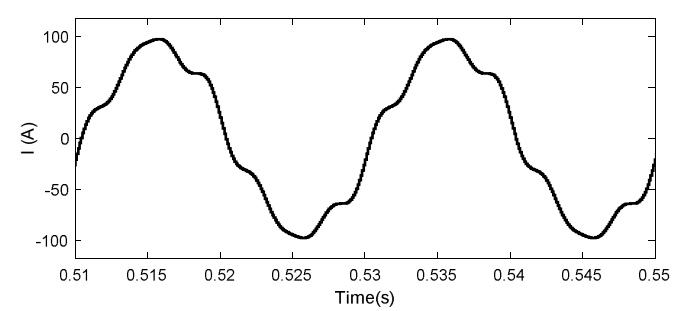

Fig. 10. Substation bus current waveform for the studied case

\section{Simulation Results}

In order to examine the effectiveness of the proposed method for harmonic elimination, it is applied to the OpenUPQC of the grid shown in Fig. 9. Furthermore, the dynamic behavior of the controller is tested through different disturbances during the simulation study. It should be noted that the simulations are carried out for 20 seconds using Matlab/SIMULINK with respect to the given description and the data given in Table III.

TABLE III. SIMULATION DATA FOR THE STUDIED CASE

\begin{tabular}{|c|c|c|}
\hline Description & Value & Unit \\
\hline Upper boundary of dead-band for PI input & 5 & - \\
\hline Lower boundary of dead-band for PI input & 4 & - \\
\hline PI integrator saturation & \pm 30 & $\mathrm{~A}$ \\
\hline PI saturation & \pm 50 & $\mathrm{~A}$ \\
\hline Discrete control output period & 0.5 & $\mathrm{~s}$ \\
\hline Telecommunication delay & 1 & $\mathrm{~s}$ \\
\hline
\end{tabular}

In order to evaluate the system response to the harmonic current deviation, the nonlinear loads applied to the grid at $t=0.5 \mathrm{~s}$ are $25 \%$ decreased at $t=8 \mathrm{~s}$. Furthermore, these loads are increased to reach $25 \%$ higher values comparing to the primary grid at $t=10 \mathrm{~s}$. The state of linear and nonlinear loads during the simulations is depicted in Fig. 11, and it is given with respect to the load values in the base grid described in Section IV.

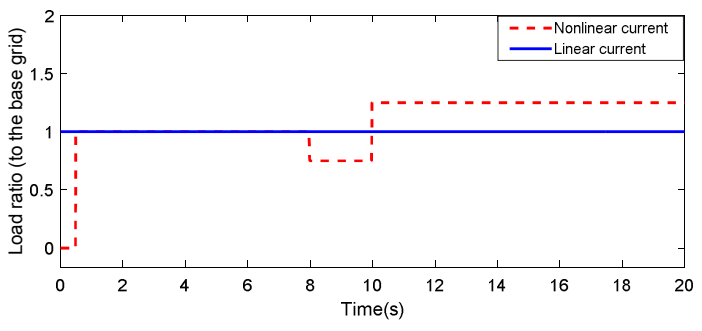

Fig. 11. Load deviation in the grid 
It should be noted that all of the linear loads of the grid are maintained constant during the simulation. Furthermore, all of the load deviations are applied a few cycles before the specified times to monitor the response of the control system to the disturbances on the determined moments.

The THD level of the current measured in the substation in presence of the control strategy is given in Fig. 12 and it is compared with the value of THD in the grid without the central controller. According to Fig. 12, when the nonlinear loads are added to the grid at $t=0.5 \mathrm{~s}$ and the THD level of the substation current is increased to $10.71 \%$, the central controller starts to update the shunt units reference currents. Due to the delay in the communication system, this command is received and applied by the shunt units at $t=1.5 \mathrm{~s}$. By a few steps, the THD reaches to $3.72 \%$ at $t=5 \mathrm{~s}$ and the control output remains constant after that. Due to the communication delay, it can be seen in Fig. 12 that the THD level decreases to $2.98 \%$ through two other steps till $t=6 \mathrm{~s}$.

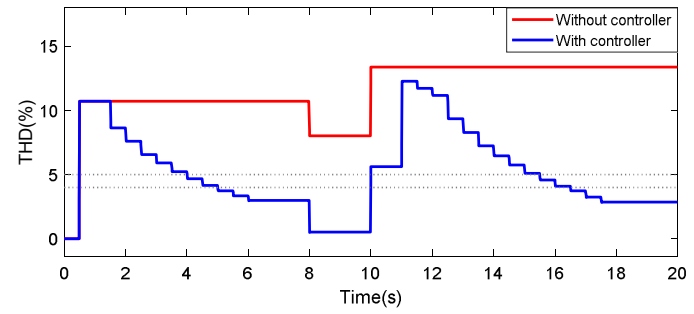

Fig. 12. The comparison of THD levels in substation current of the main grid with the grid in presence of the coordinated controller

At $t=8 \mathrm{~s}$, in presence of lower nonlinear loads in the grid, the THD level of the substation current is decreased. On the other hand, at $t=10 \mathrm{~s}$, the nonlinear loads are increased and the THD level crosses the upper boundary of the controller and the control output is updated with respect to the new measured values. But due to the delay in the communication system, the updated values are received by the shunt units at $t=11 \mathrm{~s}$. Hence, the shunt units are working with the previous output of the central controller for the time interval between $t=10 \mathrm{~s}$ and $t=11 \mathrm{~s}$. Accordingly, the curve for the THD of the main grid does not conflate to the one in presence of the controller in this period of time. After $t=11 \mathrm{~s}$, similar to the $1 \mathrm{st}$ case at $t=0.5 \mathrm{~s}$, the controller updates the output in a few steps and the THD level reaches to $3.75 \%$ in $t=16.5 \mathrm{~s}$. Then due to the delay, it decreases to $2.85 \%$ in two other time-steps till $t=17.5 \mathrm{~s}$. The waveform of the substation current in presence of the coordinated controller is compared with the main grid at $t=18 \mathrm{~s}$ in Fig. 13 .

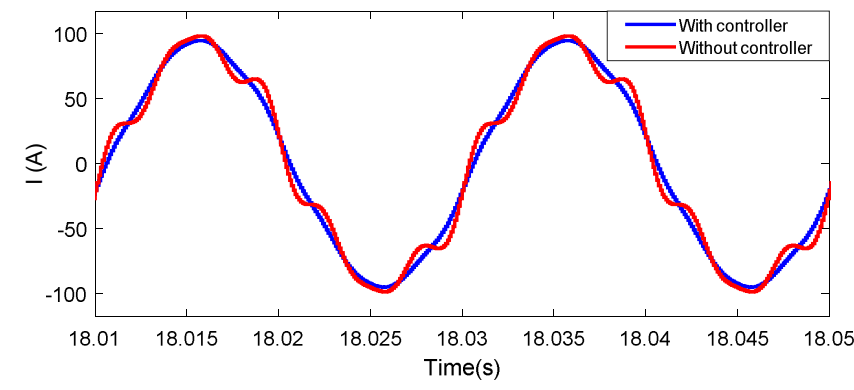

Fig. 13. Comparison of the substation current waveform in presence of the coordinated controller with waveform of the main grid

\section{CONCLUSION}

In this paper, a new coordination strategy is proposed for Open-UPQC in order to provide effective harmonic mitigation in the grid. In the first step of the proposed strategy, the harmonic currents due to the loads with no local shunt units are measured by the series unit in the substation of the feeder. With respect to the pre-defined boundaries for the allowed THD level and the dead-band considered for the controller, the central control unit decides how to provide the harmonic currents using the available capacity of shunt units inside the grid. According to the results, the proposed control strategy can provide effective harmonic elimination throughout the grid using the coordinated control of the shunt units. It should be noted that such an improvement is provided using the available capacity of the shunt units which is delivered to the DSO to be controlled by the series unit. Accordingly, the customers should be paid by the DSO for participating in the coordinated harmonic elimination in the grid. As a result, the same customers also benefit from the higher quality of the power provided by the DSO using the coordination strategy. Hence, such a method can be regarded as a feasible solution for harmonic mitigation in microgrids and modern distribution systems.

\section{REFERENCES}

[1] M. S. Rad, M. Kazerooni, M. J. Ghorbany, and H. Mokhtari, "Analysis of the grid harmonics and their impacts on distribution transformers," in 2012 IEEE Power and Energy Conference at Illinois, 2012, pp. 1-5.

[2] P. Donolo, G. Bossio, C. De Angelo, G. García, and M. Donolo, "Voltage unbalance and harmonic distortion effects on induction motor power, torque and vibrations," Electr. Power Syst. Res., vol. 140, pp. 866-873, 2016.

[3] S. M. Halpin, "Comparison of IEEE and IEC harmonic standards," in Power Engineering Society General Meeting, 2005. IEEE, 2005, pp. 2214-2216.

[4] F. G. Montoya, A. García-Cruz, M. G. Montoya, and F. ManzanoAgugliaro, "Power quality techniques research worldwide: A review," Renew. Sustain. Energy Rev., vol. 54, pp. 846-856, 2016.

[5] E. Hossain, M. R. Tür, S. Padmanaban, S. Ay, and I. Khan, "Analysis and Mitigation of Power Quality Issues in Distributed Generation Systems Using Custom Power Devices," IEEE Access, vol. 6, pp. 16816-16833, 2018.

[6] M. Farhadi-Kangarlu, E. Babaei, and F. Blaabjerg, "A comprehensive review of dynamic voltage restorers," Int. J. Electr. Power Energy Syst., vol. 92, pp. 136-155, 2017.

[7] B. Singh, S. K. Dube, and S. R. Arya, "An improved control algorithm of DSTATCOM for power quality improvement," Int. J. Electr. Power Energy Syst., vol. 64, pp. 493-504, 2015.

[8] Q. Xu, F. Ma, A. Luo, Z. He, and H. Xiao, "Analysis and Control of M3C-Based UPQC for Power Quality Improvement in Medium/HighVoltage Power Grid," IEEE Trans. Power Electron., vol. 31, no. 12, pp. 8182-8194, 2016.

[9] H. Hafezi and R. Faranda, "Dynamic Voltage Conditioner: A New Concept for Smart Low-Voltage Distribution Systems," IEEE Trans. Power Electron., vol. 33, no. 9, pp. 7582-7590, 2018.

[10] M. Brenna, R. Faranda, and E. Tironi, "A new proposal for power quality and custom power improvement: OPEN UPQC," IEEE Trans. Power Deliv., vol. 24, no. 4, pp. 2107-2116, 2009.

[11] H. Hafezi and R. Faranda, "Open UPQC series and shunt units cooperation within Smart LV Grid," in Clean Electrical Power (ICCEP), 2017 6th International Conference on, 2017, pp. 304-310.

[12] H. Hafezi, G. D’Antona, A. Dedè, D. Della Giustina, R. Faranda, and G. Massa, "Power quality conditioning in LV distribution networks: Results by field demonstration," IEEE Trans. Smart Grid, vol. 8, no. 1, pp. 418-427, 2017. 